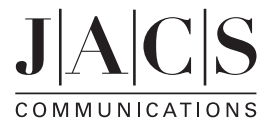

Published on Web 11/30/2007

\title{
Ordering of Dipeptide Chains on Cu Surfaces through 2D Cocrystallization

\author{
Yeliang Wang, ${ }^{*}, \dagger$ Magalí Lingenfelder, ${ }^{\dagger}$ Thomas Classen, ${ }^{\dagger}$ Giovanni Costantini, ${ }^{*}, \dagger, \S$ and Klaus Kern ${ }^{\dagger, \ddagger}$ \\ Max-Planck-Institut für Festkörperforschung, Heisenbergstrasse 1, D -70569 Stuttgart, Germany, and Institut de \\ Physique des Nanostructures, Ecole Polytechnique Fédérale de Lausanne, CH-1015 Lausanne, Switzerland
}

Received July 29, 2007; E-mail: y.wang@fkf.mpg.de; gio@fkf.mpg.de

The fabrication of novel $2 \mathrm{D}^{1 \mathrm{a}, \mathrm{b}}$ and $3 \mathrm{D}^{1 \mathrm{c}}$ molecular nanoarchitectures is attracting increasing attention in various research fields ranging from materials science to nanotechnology. Among biomolecules, peptides are very favorable building blocks, owing to the ease of their synthesis, relative stability, and chemical and biological functionalization. ${ }^{2}$ They have been used for the design and construction of nanostructures for diverse applications, such as templates for the growth of functional networks, ${ }^{3}$ biosensors for monitoring enzymatic reactions, ${ }^{4}$ and organic catalysts for asymmetric aldol reactions. ${ }^{5}$ A large amount of ordered peptide nanostructures with different geometries, ${ }^{6}$ including nanotubes, nanospheres and nanofilaments, have been produced in solution or in vacuum. Peptide monolayer structures self-assembled on solid surfaces typically show a strong tendency to form chains. ${ }^{6 c-e}$ Two-dimensional (2D) extended arrangements are difficult to produce due to a pronounced anisotropy in the intermolecular interactions.

Here we report on the ordering and interconnection of 1D dipeptide nanostructures. Individual diphenylalanine molecules (Phe-Phe, Figure 1), only form short isolated chains with a broad length distribution when deposited on $\mathrm{Cu}$ substrates. By exploiting 2D cocrystallization with the organic linker terephthalic acid (TPA), we show that continuous and highly periodic dipeptide arrangements can be formed on both the anisotropic $\mathrm{Cu}(110)$ and the isotropic $\mathrm{Cu}(100)$ surface. This approach might be extended to the fabrication of similar peptide-based nanostructures with potential applications in biocompatible functional surfaces.

Scanning tunneling microscopy (STM) measurements of L-PheL-Phe molecules deposited under ultrahigh vacuum on $\mathrm{Cu}$ surfaces reveal a preferential self-organization in the form of $1 \mathrm{D}$ chains. On the $\mathrm{Cu}(110)$ surface the Phe-Phe chains are typically isolated and characterized by a high density of kinks. On $\mathrm{Cu}(100)$ the chains show four possible orientations and are typically shorter, and their distribution is similarly dispersed (see Figure S1 in the Supporting Information).

The formation of isolated chains suggests different intra- and interchain interactions. The binding between Phe-Phe molecules, which results in the development of supramolecular chains, is most probably due to an interaction between the carboxylic group of one molecule and the amino group of the neighboring one. ${ }^{7}$ On the other hand, a nonperfect matching of the chain structures with the underlying substrate might cause the frequent kink defects. The same mismatch could also generate a substrate-mediated repulsion among the chains and, similarly to what is observed in other systems, ${ }^{8}$ result in their separation. Chain-chain repulsion and kink defects are the reasons why extended and ordered structures are never formed, independently of the molecular coverage (see Figure $\mathrm{S} 1)$.

\footnotetext{
$\dagger$ Max-Planck-Institut für Festkörperforschung.

Ecole Polytechnique Fédérale de Lausanne.

\& Present address: Department of Chemistry, University of Warwick, Coventry CV4 7AL, U.K.
}

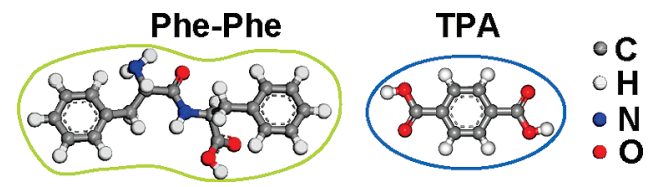

Figure 1. Chemical structure of di-L-phenylalanine (L-Phe-L-Phe) and terephthalic acid (TPA).

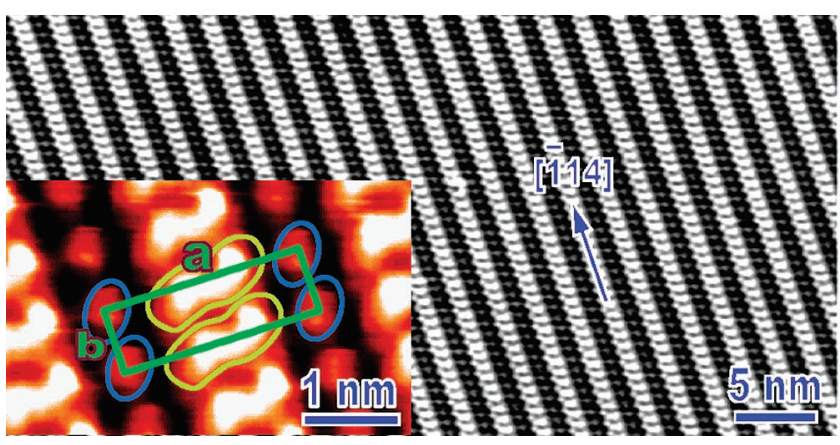

Figure 2. STM images of $2 \mathrm{D}$ extended L-Phe-L-Phe/TPA hybrid motif on $\mathrm{Cu}(110)$. Phe-Phe rows appear brighter than TPA rows. The quadrangle in the inset marks one unit of the molecular superlattice. The ellipse and dumbbell indicate TPA and Phe-Phe molecules, respectively. The images are acquired at $-2.0 \mathrm{~V}, 0.5 \mathrm{nA}$ for the large-scale one and at $-1.0 \mathrm{~V}, 0.5$ $\mathrm{nA}$ for the high-resolution one.

In order to overcome this limitation, we have co-deposited a molecular linker (TPA) with the aim of connecting the isolated Phe-Phe chains by effectively enhancing their interaction. This kind of multicomponent method is similar to the procedure used in the fabrication of 3D cocrystals, ${ }^{9}$ and first attempts have recently been made also in 2D. ${ }^{10}$ Following this approach, it was shown that molecules characterized by a $1 \mathrm{D}$ arrangement in their singlecomponent phase are able to form 3D cocrystals stabilized by hydrogen bonds when mixed with specific linker molecules. ${ }^{9}$

After co-depositing Phe-Phe and TPA, 2D ordering and extending of supramolecular structures emerge on both copper surfaces. Our STM measurements show that these highly ordered motifs extend over large domains (up to $150 \times 150 \mathrm{~nm}^{2}$ ) and are stable up to temperatures of $540 \mathrm{~K}$. For both substrates the molecules self-organize with a specific stoichiometry of Phe-Phe to TPA (1:1). This ratio is self-selected and is retained even for an excess of the initial Phe-Phe component (see Figure S2). This indicates that these nanoarchitectures are easy to produce, since their formation does not require a precise control of the relative amount of the deposited components. Besides inducing the formation of extended domains, the TPA linker improves the regularity of the Phe-Phe chains by lifting the kink defects. Moreover, on $\mathrm{Cu}(100)$ only two main chain orientations are left out of the original four possible directions. Other binary molecular systems produce either segregated monolayers or new motifs presenting a multiphase behavior where the ratio of the components must be carefully controlled. ${ }^{10 \mathrm{a}-\mathrm{c}}$ The strength of the $2 \mathrm{D}$ cocrystallization method 


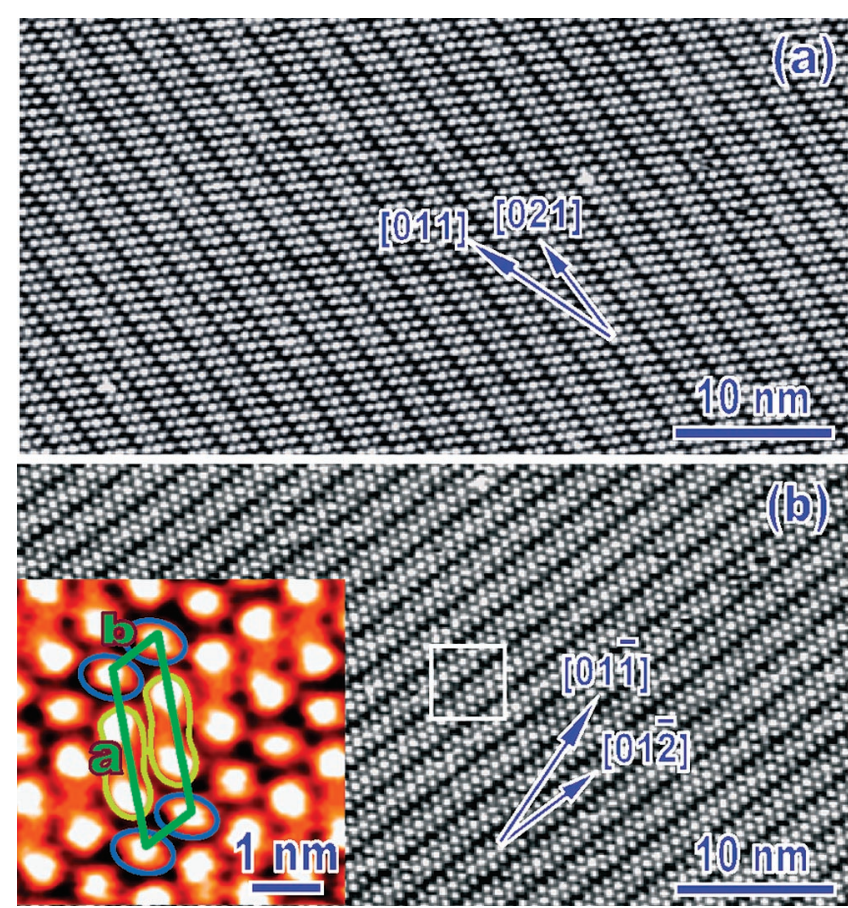

Figure 3. STM images of $2 \mathrm{D}$ extended L-Phe-L-Phe/TPA motifs on $\mathrm{Cu}$ (100). Domains in (a) and (b) show a $90^{\circ}$ rotation symmetry. The rectangle in (b) marks the area shown as a close-up image in the inset. All images are recorded at $-1.26 \mathrm{~V}, 0.5 \mathrm{nA}$.

presented here lies in the capability to improve the order and the extension of a preexisting molecular structure with a self-selected stoichiometry.

The STM image in Figure 2 shows a well-ordered L-Phe-LPhe/TPA hybrid molecular film on $\mathrm{Cu}(110)$. One supramolecular unit cell ( $\left.a=24.1 \AA, b=7.7 \AA, \theta_{a-b}=87.9^{\circ}\right)$ is superimposed on the high-resolution image in the inset. The superstructure can be described by the matrix $\left(\begin{array}{l}9 \\ 1-2\end{array}\right)$ with respect to the substrate [1 $\left.\overline{10}\right]$ and [001] directions. The brighter Phe-Phe rows combine alternately with the dimmer TPA rows and extend periodically over the substrate. Within this hybrid arrangement the Phe-Phe chains keep the identical [114] direction they had in the single-component phase. Clearly, the effect of the TPA linkers is to combine and extend the isolated dipeptide chains without altering their inherent structure nor modifying the final surface chirality.

Figure 3 shows two large-scale chiral L-Phe-L-Phe/TPA hybrid domains with a $90^{\circ}$ relative rotation observed on $\mathrm{Cu}(100)$. The supramolecular structures can be denoted by the matrices $\left(\begin{array}{rr}-7 & 7 \\ 3 & 1\end{array}\right)$ and $\left(\begin{array}{ll}-7 & -7 \\ -1 & 3\end{array}\right)$ relative to the substrate principal [011] and [011] directions. Also here the chains keep the same orientations ([021] and [01 $\overline{2}]$ ) as in the pure L-Phe-L-Phe phase, resulting in a surface with local organizational chirality. ${ }^{11 \mathrm{a}}$

The ability of the TPA linker molecule to order and lengthen the discrete dipeptide chains evidently does not depend on the different substrate geometry. This suggests that also in the 2D case, the cocrystallization approach might be sufficiently robust and of general applicability.

The close spacing among the molecules and their orientation indicate that intermolecular $\mathrm{H}$-bonding might be the main driving force for the formation of the final ordered structures. ${ }^{9,10 b-g, 11}$ Moreover, the integrity of the Phe-Phe chain motifs indicates that the intrachain binding is stronger than the TPA-chain interaction. However, this latter interaction is evidently strong enough to overcome the original chain-chain repulsion and to avoid the formation of kink defects. This, together with the manifestation of a $2 \mathrm{D}$ cocrystallization instead of a phase separation, suggests the following hierarchy in the intermolecular binding strengths: intrachain > chain-TPA > TPA-TPA.

In summary, we have demonstrated the possibility of fabricating extended surface-supported dipeptide architectures by the 2D cocrystallization method. The organic linker TPA was used as a molecular "glue" to bridge isolated Phe-Phe chains and to remarkably improve their regularity. The formation of highly periodic motifs appears to be independent of the specific substrate structure. The characteristics of Phe-Phe molecular chains are successfully transferred to the final structures which, albeit being 2D extended, retain a $1 \mathrm{D}$ chiral modulation. Besides acting as nanotemplates, these substrates might therefore be interesting for heterogeneous enantioselective catalysis applications. ${ }^{11} \mathrm{We}$ speculate that the approach presented here might be extended to a wider range of biomolecules with increasing functional groups and could be used for the construction of surface-supported organic cocrystals. Experiments combining different types of polypeptides and linkers are underway.

Acknowledgment. We gratefully acknowledge N. Lin and L. S. Wang for fruitful discussions. Y. Wang acknowledges the Humboldt Foundation for financial support.

Supporting Information Available: Experimental details and STM data of pure Phe-Phe chains on $\mathrm{Cu}(110)$ and $\mathrm{Cu}(100)$ surfaces. This material is available free of charge via the Internet at http://pubs.acs.org.

\section{References}

(1) (a) Barth, J. V.; Costantini, G.; Kern, K. Nature 2005, 437, 671. (b) Rosei, F.; Schunack, M.; Naitoh, Y.; Jiang, P.; Gourdon, A.; Laegsgaard, E.; Stensgaard, I.; Joachim, C.; Besenbacher, F. Prog. Surf. Sci. 2003, 71 , 95. (c) Crespo-Biel, O.; Ravoo, B. J.; Reinhoudt, D. N.; Huskens, J. J. Mater. Chem. 2006, 16, 3997.

(2) (a) Colombo, G.; Soto, P.; Gazit, E. Trends Biotechnol. 2007, 25, 211 (b) Zhao, X.; Zhang, S. Macromol. Biosci. 2007, 7, 13

(3) Silva, G. A.; Czeisler, C.; Niece, K. L.; Beniash, E.; Harrington, D. A.; Kessler, J. A.; Stupp, S. I. Science 2004, 303, 1352.

(4) Zhao, Z.; Banerjee, I. A.; Matsui, H. J. J. Am. Chem. Soc. 2005, 127, 8930.

(5) Tsogoeva, S. B.; Wei, S. Tetrahedron: Asymmetry 2005, 16, 1947.

(6) (a) Williams, B. A. R.; Lund, K.; Liu, Y.; Yan, H.; Chaput, J. C. Angew. Chem., Int. Ed. 2007, 46, 3051. (b) Gong, J. R.; Yan, H. J.; Yuan, Q. H.; Xu, L. P.; Bo, Z. S.; Wan, L. J. J. Am. Chem. Soc. 2006, 128, 12384. (c) Whitehouse, C.; Fang, J.; Aggeli, A.; Bell, M.; Brydson, R.; Fishwick, C. W. G.; Henderson, J. R.; Knobler, C. M.; Owens, R. W.; Thomson, N. H.; Smith, D. A.; Boden, N. Angew. Chem., Int. Ed. 2005, 44, 1965. (d) Stensgaard, I. Surf. Sci. Lett. 2003, 545, 747. (e) Barlow, S. M.; Haq, S.; Raval, R. Langmuir 2001, 17, 3292.

(7) Lingenfelder, M.; Tomba, G.; Costantini, G.; Colombi Ciacchi, L.; De Vita, A.; Kern, K. Angew. Chem., Int. Ed. 2007, 46, 4492.

(8) (a) Barth, J. V.; Weckesser, J.; Cai, C.; Günter, P.; Bürgi, L.; Jeandupeux, O.; Kern, K. Angew. Chem., Int. Ed. 2000, 39, 1230. (b) Kern, K.; Niehus, H.; Schatz, A.; Zeppenfeld, P.; George, J.; Comsa, G. Phys. Rev. Lett. 1991, 67, 855.

(9) (a) Wiechert, D.; Mootz, D.; Dahlems, T. J. Am. Chem. Soc. 1997, 119, 12665. (b) Goroff, N. S.; Curtis, S. M.; Webb, J. A.; Fowler, F. W.; Lauher, J. W. Org. Lett. 2005, 7, 1891 .

(10) (a) de Wild, M.; Berner, S.; Suzuki, H.; Yanagi, H.; Schlettwein, D.; Ivan, S.; Baratoff, A.; Guentherodt, H. J.; Jung, T. A. ChemPhysChem 2002, 3, 881. (b) Theobald, J. A.; Oxtoby, N. S.; Phillips, M. A.; Champness, N. R.; Beton, P. H. Nature 2003, 424, 1029. (c) Xu, B.; Tao, C.; Williams, E. D.; Reutt-Robey, J. E. J. Am. Chem. Soc. 2006, 128, 8493. (d) CañasVentura, M. E.; Xiao, W.; Wasserfallen, D.; Müllen, K.; Brune, H.; Barth, J. V.; Fasel, R. Angew. Chem., Int. Ed. 2007, 46, 1814. (e) Nath, K. G.; Ivasenko, O.; Miwa, J. A; Dang, H.: Wuest, J. D.; Nanci, A.; Perepichka, D. F.; Rosei, F. J. Am. Chem. Soc. 2006, 128, 4212. (f) Tao, F.; Bernasek, S. L. Surf. Sci. 2007, 601, 2284. (g) Tao, F.; Bernasek, S. L. J. Am. Chem Soc. 2005, 127, 12750. (h) Hipps, K. W.; Scudiero, L.; Barlow, D. E. Cooke, M. P. J. Am. Chem. Soc. 2002, 124, 2126. (i) Wei, Y.; Tong, W.; Wise, C.; Wei, X.; Armbrust, K.; Zimmt, M. J. Am. Chem. Soc. 2006, 128,13362

(11) (a) Ortega Lorenzo, M.; Baddeley, C. J.; Muryn, C.; Raval, R. Nature 2000, 404, 376. (b) Chen, Q.; Richardson, N. V. Nat. Mater. 2003, 2 324. (c) Cai, Y.; Bernasek, S. L. J. Am. Chem. Soc. 2004, 126, 14234 JA075118V 